\title{
A utilização de métodos não farmacológicos para o alívio da dor durante o trabalho de parto e parto
}

\author{
The use of non-pharmacological methods for pain relief during childbirth and childbirth \\ work
El uso de métodos no farmacológicos para el alivio del dolor durante el nacimiento y el trabajo del nacimiento

Graziela Santos da França', Catarina Maria Lima1, Thamires Lima Sarah¹, Gabriela Romão de Almeida Carvalho Santos ${ }^{2 *}$, Letícia Loss de Oliveira ${ }^{3}$, Rodrigo Rocha de Souza ${ }^{4}$.

\section{RESUMO}

Objetivo: identificar quais são os métodos não farmacológicos utilizados para alívio da dor durante o trabalho de parto e parto. Métodos: Trata-se de uma revisão integrativa realizada nas bases de dados Scientific Electronic Library Online (SciELO) e Pubmed, por meio dos Descritores em Ciências Saúde: "Dor do parto", "Terapias complementares" e "Trabalho de parto" e os Medical Subject Headings: "Labor pain", "Complementary therapy" e "Labor". Os critérios de inclusão foram artigos nos idiomas português, inglês ou espanhol, com texto completo, que abordassem a temática, de 2015 a 2020. Como critérios de exclusão, artigos repetidos nas bases de dados, incompletos e fora do período estipulado, sendo selecionados 30 estudos. Resultados: Identificou-se inúmeros métodos não farmacológicos para alívio da dor durante o trabalho de parto e parto, entre eles auriculoterapia, acupressão, acupuntura, aromaterapia, musicoterapia e massagens. Considerações finais: Através desse estudo identificou-se quais são os métodos não farmacológicos utilizados para alívio da dor no trabalho de parto e parto, verificando a eficácia desses métodos, sendo eles diversificados e com baixo custo.

Palavras-chave: Dor do parto, Terapias complementares, Trabalho de parto.

\begin{abstract}
Objective: To identify which are the non-pharmacological methods used for pain relief during labor. Methods: This is an integrative review based on data from the Scientific Electronic Library Online (SciELO) and PubMed, with Health Sciences Descriptors: "Labor pain", "Complementary therapies" and "Labor". As inclusion criteria we have articles published in full from 2015 to 2020, in Portuguese, English or Spanish, addressing the theme. The exclusion criteria: articles repeated in the database, incomplete or out of date, 30 studies were selected to compose the review. Results: Several nonpharmacological methods for pain relief during labor were identified, including: auriculotherapy, shower bath, use of therapeutic ball, acupressure, acupuncture, electroacupuncture, aromatherapy, music therapy, ice massage at Hoku point or in the L4 region, sacral and lumbar massage, reflexology technique, yoga during pregnancy, hypnosis, breathing techniques and the rebozo technique. Final considerations: This study identified which are the non-pharmacological methods used to relieve the pain during labor and delivery, proving the effectiveness of these methods, being them diversified and with low cost.
\end{abstract}

Key words: Labor pain, Complementary therapy, Labor obstetric.

\section{RESUMÉN}

Objetivo: Identificar cuáles son los métodos no farmacológicos utilizados para alivio del dolor durante el trabajo de parto y parto. Métodos: se trata de una revisión integradora realizada en bases de datos Scientific Electronic Library Online (SciELO) y Pubmed, por medio de los Descriptores en Ciencias de la Salud (DeCS): "Dolor del parto", "Terapias complementares" y "Trabajo de parto" y los Medical Subject Headings: "Labor pain", "Complementary therapy" y "Labor". En los criterios de inclusión fueron considerados artículos en los idiomas de portugués, inglés o español, con texto completo, que tratasen la temática, de 2015 a 2020. Como criterios de exclusión, artículos repetidos en bases de datos, incompletos y fuera del periodo estipulado, siendo seleccionados 30 estudios científicos para formar parte del análisis. Resultados: fueron identificados innúmeros métodos no farmacológicos para alivio del dolor durante el parto y parto, entre ellos, auriculoterapia, baño de ducha, utilización de pelota, acupresión, acupuntura, eletroacupuntura, aromaterapia, musicoterapia, masaje con hielo en el punto Hoku o en el LI4, masaje sacro lumbar, técnica de reflexología y yoga durante

\footnotetext{
${ }^{1}$ Centro Universitário IBMR, Rio de Janeiro - RJ.

2 Universidade Salvador (UNIFACS), Salvador - BA. *E-mail: gabrielaromaoalmeida98@gmail.com

3 Universidade do Estado do Rio de Janeiro (UERJ), Rio de Janeiro - RJ.

${ }^{4}$ Universidade Federal Fluminense (UFF), Rio de Janeiro - RJ.
} 
el embarazo, hipnosis, técnicas con respiración con inspiración y espiración profunda y técnica del Rebozo. Consideraciones finales: Mediante este estudio se identificó cuáles son los métodos no farmacológicos utilizados para aliviar el dolor en el trabajo de parto y el parto, verificando la eficacia de estos métodos, siendo ellos diversificados y con bajo costo.

Palabras clave: Dolor de parto, Terapias complementarias, Trabajo de parto.

\section{INTRODUÇÃO}

O trabalho de parto e o parto são momentos biopsicossociais, que constituem um processo de transição, no qual a mulher passa a ser mãe, sendo um momento permeado por valores afetivos, emocionais, sociais e culturais. Entretanto, além de ser um momento marcado por inúmeros sentimentos, a mulher em trabalho de parto sofre influências hormonais, no qual ocorre liberação de ocitocina, que é o hormônio responsável por causar contratilidade uterina, gerando estímulos dolorosos durante o trabalho de parto e parto (MAZONI SR, et al., 2013; HALL JE, 2015).

Apesar do parto se configurar como uma das causas mais comuns de dor aguda, o nível da dor pode variar de acordo com fatores emocionais, ambientais, existenciais, culturais, pessoais e a forma como a dor é percebida. Isto posto, durante o parto, na presença de dor aguda, as mulheres podem manifestar alterações fisiológicas, entre elas, a elevação da pressão arterial, da frequência cardíaca e respiratória, além de estresse e ansiedade, ocasionando em uma sobrecarga dos sistemas corporais, afetando o trabalho de parto (HALL JE, 2015).

Dessa forma, intervenções que diminuam os efeitos causados pela dor durante o trabalho de parto e parto e que elevem a tolerância da mulher à dor durante o nascimento do bebê são necessárias e de fundamental importância para promover conforto, qualidade e segurança no parto (MAZONI SR, et al., 2013).

Porém, com a institucionalização do parto, essas intervenções passaram a ser guiadas pela técnica, pela tecnologia e pela medicalização. Além disso, o uso irracional de instrumentos e medicamentos e a realização de intervenções desnecessárias são fatores que dificultam a redução da mortalidade materna e perinatal, contribuindo para fragmentação da assistência à mulher (MELO PDS, et al., 2020).

Frequentemente, a utilização de métodos invasivos, elementos estimuladores e intervenções desnecessárias elevam a dor. Todavia, a liberdade de movimento, a presença do acompanhante e o emprego de métodos não farmacológicos tem relação ao maior conforto e redução da dor durante o trabalho de parto e parto. Dessa forma, os métodos não farmacológicos surgem como uma alternativa para aliviar as dores da forma mais natural possível, tornando as mulheres protagonistas desse momento e tornando esse processo um marco para a mulher e sua família (HALL JE, 2015).

Com base nessas reflexões o presente estudo será conduzido pela seguinte pergunta norteadora: "Quais são os métodos não farmacológicos utilizados para alívio da dor durante o trabalho de parto e no parto relatados nos últimos 5 anos?". Diante disso, o estudo possui o objetivo de identificar quais são os métodos não farmacológicos utilizados para alívio da dor durante o trabalho de parto e parto.

\section{MÉTODOS}

Trata-se de uma revisão de literatura do tipo integrativa. Para que a revisão integrativa possa ser realizada, são exigidas seis etapas: Identificação do tema e seleção da questão de pesquisa; Estabelecimento dos critérios de elegibilidade; Identificação dos estudos nas bases científicas; Avaliação dos estudos selecionados e análise crítica; Categorização dos estudos, avaliação e interpretação dos resultados; Apresentação da revisão integrativa (BOTELHO LLR, et al., 2011).

O levantamento dos artigos realizou-se entre os meses de agosto e setembro de 2020, através das bases de dados Scientific Electronic Library Online (SciELO) e Pubmed. A partir dos Descritores em Ciências Saúde (DeCS): "Dor do parto", "Terapias complementares" e "Trabalho de parto" e os Medical Subject Headings (MeSH): "Labor pain", "Complementary therapy" and "Labor". Os descritores foram cruzados através do operador booleano "AND" para busca simultânea dos assuntos. 
Para realizar a busca e seleção dos artigos foram considerados os seguintes critérios de inclusão: artigos nos idiomas português, inglês ou espanhol, com texto completo disponível e que abordassem sobre os métodos não farmacológicos utilizados no alívio da dor do parto, no período de 2015 a 2020. E, como critérios de exclusão, foram considerados os artigos repetidos nas bases de dados, sendo mantidos em apenas uma base, artigos incompletos, revisões de literatura, teses, dissertações, capítulos de livro, estudos que não abordassem sobre os métodos não farmacológicos utilizados no alívio da dor do parto e fora do período estipulado.

A partir dessa busca, foram encontrados 421 estudos. Após leitura pareada dos títulos e resumos para constatar quais se adequaram aos critérios de inclusão e exclusão estabelecidos, 6 foram excluídos por não estarem disponíveis na íntegra, 118 foram excluídos por não abordarem a temática do estudo, 144 foram excluídos por estarem fora do período estabelecido, 7 foram excluídos por estarem repetidos nas bases de dados, sendo mantidos em apenas uma base e 116 foram excluídos por serem revisões, sendo selecionados 30 estudos para compor o presente estudo (Figura 1).

Figura 1 - Fluxograma de resultados das buscas nas bases de dados.

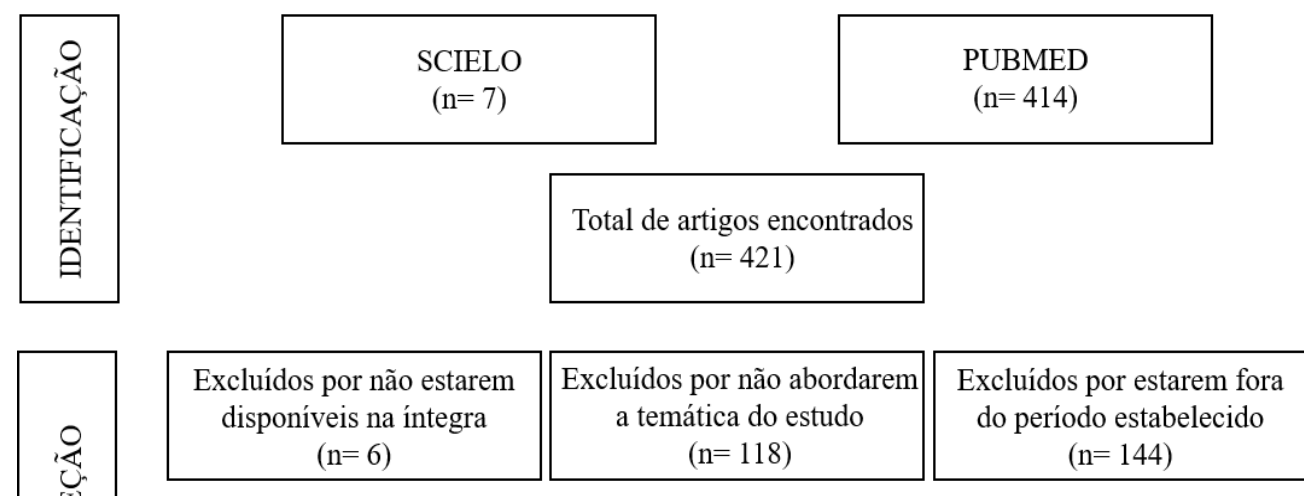

\begin{tabular}{|c|c|}
$\begin{array}{c}\text { Excluídos por estarem } \\
\text { repetidos nas bases de dados } \\
(\mathrm{n}=7)\end{array}$ & $\begin{array}{c}\text { Excluídos por serem } \\
\text { revisões de literatura } \\
(\mathrm{n}=116)\end{array}$ \\
\hline
\end{tabular}

\begin{tabular}{|c|c|}
\hline $\begin{array}{c}\text { Artigos selecionados para } \\
\text { leitura na íntegra } \\
(\mathrm{n}=30)\end{array}$ \\
\hline \\
\hline \\
\hline
\end{tabular}

Fonte: França GSD, et al., 2021.

A análise de dados foi realizada por meio de um banco de dados montado no programa Excel versão 2016, no qual os mesmos foram convertidos e apresentados em forma de resultados, com o intuito de demonstrar os resultados obtidos, atendendo aos objetivos da pesquisa. Tais resultados foram confrontados com as evidências disponíveis na literatura e discutidos com o embasamento científico.

Por ter como referência bases públicas, não foi necessária a submissão ao Comitê de Ética e Pesquisa, respeitando, porém, os preceitos éticos estabelecidos na resolução número 466/2012 do Conselho Nacional de Saúde.

\section{RESULTADOS}

Após a análise dos artigos, obteve-se como resultado final 30 estudos, no qual as principais informações foram agrupadas (Quadro 1). 
Quadro 1 - Distribuição dos artigos incluídos na revisão segundo título, autor e ano de publicação, objetivo, tipo de pesquisa e resultados.

\begin{tabular}{|c|c|c|c|c|}
\hline Título & Autor/ano & Objetivo & Tipo de estudo & Resultado \\
\hline $\begin{array}{l}\text { Efetividade da } \\
\text { auriculoterapia sobre a dor } \\
\text { no trabalho de parto: ensaio } \\
\text { clínico randomizado }\end{array}$ & $\begin{array}{l}\text { MAFETONI RR, } \\
\text { et al., } 2019\end{array}$ & $\begin{array}{l}\text { Avaliar a efetividade da } \\
\text { auriculoterapia sobre a dor na fase } \\
\text { ativa do trabalho de parto. }\end{array}$ & $\begin{array}{l}\text { Ensaio clínico } \\
\text { randomizado }\end{array}$ & $\begin{array}{l}\text { As parturientes que receberam auriculoterapia } \\
\text { durante o trabalho de parto mostraram } \\
\text { redução na intensidade da dor. }\end{array}$ \\
\hline $\begin{array}{l}\text { Terapias complementares no } \\
\text { trabalho de parto: ensaio } \\
\text { clínico randomizado }\end{array}$ & $\begin{array}{l}\text { CAVALCANTI } \\
\text { ACV, et al., } 2019\end{array}$ & $\begin{array}{l}\text { Avaliar o efeito do banho quente de } \\
\text { chuveiro e exercício perineal com } \\
\text { bola suíça isolados e de forma } \\
\text { combinada, sobre a percepção da } \\
\text { dor, ansiedade e progressão do } \\
\text { trabalho de parto. }\end{array}$ & $\begin{array}{l}\text { Ensaio clínico } \\
\text { randomizado }\end{array}$ & $\begin{array}{l}\text { Houve aumento no escore de dor e redução } \\
\text { da ansiedade em todos os grupos, sobretudo } \\
\text { quando utilizaram banho de chuveiro. }\end{array}$ \\
\hline $\begin{array}{l}\text { Os efeitos da acupressão } \\
\text { nas dores do parto durante o } \\
\text { parto: ensaio clínico } \\
\text { randomizado }\end{array}$ & $\begin{array}{l}\text { MAFETONI RR e } \\
\text { SHIMO AKK } \\
2016\end{array}$ & $\begin{array}{l}\text { Analisar os efeitos da acupressão } \\
\text { no ponto sanyinjiao sobre a dor na } \\
\text { fase ativa do trabalho de parto, em } \\
\text { gestantes atendidas em } \\
\text { maternidade pública. }\end{array}$ & $\begin{array}{l}\text { Ensaio clínico } \\
\text { randomizado }\end{array}$ & $\begin{array}{c}\text { A acupressão no ponto sanyinjiao se mostrou } \\
\text { uma medida útil no alívio da dor, não invasiva } \\
\text { e um meio de melhorar a qualidade dos } \\
\text { cuidados à parturiente. }\end{array}$ \\
\hline $\begin{array}{l}\text { Parâmetros maternos e } \\
\text { perinatais após intervenções } \\
\text { não farmacológicas: um } \\
\text { ensaio clínico randomizado } \\
\text { controlado }\end{array}$ & $\begin{array}{l}\text { MELO PDS, et } \\
\text { al., } 2020\end{array}$ & $\begin{array}{l}\text { Analisar os efeitos do banho } \\
\text { quente, de exercícios perineais } \\
\text { com bola suíça ou de ambos } \\
\text { durante o trabalho de parto em } \\
\text { parâmetros maternos e perinatais. }\end{array}$ & $\begin{array}{l}\text { Ensaio clínico } \\
\text { randomizado }\end{array}$ & $\begin{array}{c}\text { As intervenções isoladas ou combinadas } \\
\text { (banho quente e exercícios perineais com bola } \\
\text { suíça) são uma forma segura de assistência } \\
\text { ao parto uma vez que elas não afetam } \\
\text { negativamente os parâmetros maternos e } \\
\text { perinatais. }\end{array}$ \\
\hline $\begin{array}{l}\text { O efeito da aromaterapia } \\
\text { com essência de lavanda na } \\
\text { gravidade da dor do parto e } \\
\text { na duração do trabalho de } \\
\text { parto em mulheres } \\
\text { primíparas }\end{array}$ & $\begin{array}{l}\text { YAZDKHASTI M } \\
\text { e PIRAK A, } 2016\end{array}$ & $\begin{array}{l}\text { Investigar o efeito da inalação da } \\
\text { essência de Lavanda na gravidade } \\
\text { da dor do parto e na duração do } \\
\text { trabalho de parto. }\end{array}$ & $\begin{array}{l}\text { Ensaio clínico } \\
\text { randomizado }\end{array}$ & $\begin{array}{l}\text { A aromaterapia com essência de lavanda } \\
\text { pode ser uma opção terapêutica eficaz. }\end{array}$ \\
\hline $\begin{array}{l}\text { Terapias complementares e } \\
\text { alternativas para aliviar a dor } \\
\text { do parto: um estudo } \\
\text { comparative entre a } \\
\text { musicoterapia e a massagem } \\
\text { com gelo Hoku Point }\end{array}$ & $\begin{array}{l}\text { DEHCHESHMEH } \\
\text { FS e RAFIEI H, } \\
2015\end{array}$ & $\begin{array}{l}\text { Comparar o efeito de dois tipos de } \\
\text { métodos não farmacológicos de } \\
\text { alívio da dor do parto } \\
\text { "musicoterapia" e "Hoku point ice } \\
\text { massage". }\end{array}$ & $\begin{array}{l}\text { Estudo prospectivo, } \\
\text { randomizado }\end{array}$ & $\begin{array}{l}\text { Após a intervenção, os escores médios de dor } \\
\text { foram significativamente menores em todos os } \\
\text { momentos. A musicoterapia e a massagem } \\
\text { com gelo no ponto Hoku são métodos de fácil } \\
\text { acesso e baratos e têm um efeito semelhante } \\
\text { no alívio da dor do parto. }\end{array}$ \\
\hline
\end{tabular}

REAS | Vol. 13(5) | DOI: https://doi.org/10.25248/REAS.e7215.2021

Página 4 de 14 


\begin{tabular}{|c|c|c|c|c|}
\hline Título & Autor/ano & Objetivo & Tipo de estudo & Resultado \\
\hline $\begin{array}{l}\text { Eficácia da aromaterapia } \\
\text { para reducir a dor durante o } \\
\text { trabalho de parto: um ensaio } \\
\text { clínico randomizado }\end{array}$ & $\begin{array}{l}\text { TANVISUT R, et } \\
\text { al., } 2018\end{array}$ & $\begin{array}{l}\text { Determinar a eficácia da } \\
\text { aromaterapia para reduzir a dor } \\
\text { durante o trabalho de parto. }\end{array}$ & $\begin{array}{l}\text { Ensaio clínico } \\
\text { randomizado }\end{array}$ & $\begin{array}{l}\text { O escore médio de dor da fase latente e ativa } \\
\text { inicial foi menor no grupo de aromaterapia. A } \\
\text { aromaterapia é útil na redução da dor na fase } \\
\text { latente e ativa inicial. }\end{array}$ \\
\hline $\begin{array}{l}\text { O efeito da reflexologia podal } \\
\text { na ansiedade, dor e } \\
\text { resultados do parto em } \\
\text { mulheres primigestas }\end{array}$ & $\begin{array}{l}\text { MOGHIMI- } \\
\text { HANJANI S, et } \\
\quad \text { al., } 2015\end{array}$ & $\begin{array}{l}\text { Revisar e determinar o efeito da } \\
\text { reflexologia podal na ansiedade, } \\
\text { dor e resultados do parto em } \\
\text { mulheres primigestas. }\end{array}$ & Estudo clínico & $\begin{array}{c}\text { A aplicação da técnica de reflexologia diminuiu } \\
\text { significativamente a intensidade da dor e a } \\
\text { duração do trabalho de parto, bem como o } \\
\text { nível de ansiedade. }\end{array}$ \\
\hline $\begin{array}{l}\text { loga durante a gravidez: os } \\
\text { efeitos na dor do parto e nos } \\
\text { resultados do parto (um } \\
\text { ensaio clínico randomizado) }\end{array}$ & $\begin{array}{c}\text { JAHDI F, et al., } \\
2017\end{array}$ & $\begin{array}{l}\text { Investigar os efeitos de um } \\
\text { programa de ioga pré-natal na } \\
\text { percepção da dor do parto } \\
\text { materno. }\end{array}$ & $\begin{array}{l}\text { Ensaio clínico } \\
\text { randomizado }\end{array}$ & $\begin{array}{l}\text { Os participantes do grupo controle relataram } \\
\text { maior intensidade de dor em comparação ao } \\
\text { grupo que realizou a ioga. }\end{array}$ \\
\hline $\begin{array}{l}\text { O efeito da massagem sacral } \\
\text { na dor do parto e na } \\
\text { ansiedade: um ensaio clínico } \\
\text { randomizado }\end{array}$ & $\begin{array}{l}\text { ÇEVIK AS e } \\
\text { KARADUMAN S, } \\
2019\end{array}$ & $\begin{array}{l}\text { Determinar o efeito da massagem } \\
\text { sacral na dor do parto e na } \\
\text { ansiedade. }\end{array}$ & $\begin{array}{l}\text { Ensaio clínico } \\
\text { randomizado }\end{array}$ & $\begin{array}{l}\text { As escalas visuais analógica do grupo } \\
\text { experimental foram estatisticamente } \\
\text { significativamente menores do que as do } \\
\text { grupo de controle, evidenciando que a } \\
\text { massagem sacral aplicada durante o trabalho } \\
\text { de parto reduziu a dor do parto. }\end{array}$ \\
\hline $\begin{array}{c}\text { Estudo de terapias } \\
\text { complementares para } \\
\text { trabalho de parto e } \\
\text { Nascimento: um ensaio } \\
\text { clínico randomizado e } \\
\text { controlado de medicina } \\
\text { integrative pré-natal para } \\
\text { controle da do rem trabalho } \\
\text { de parto }\end{array}$ & $\begin{array}{l}\text { LEVETT KM, et } \\
\text { al., } 2016\end{array}$ & $\begin{array}{l}\text { Avaliar o efeito de um programa de } \\
\text { educação em medicina integrativa } \\
\text { pré-natal, além dos cuidados } \\
\text { habituais para mulheres nulíparas, } \\
\text { sobre o uso de peridural intraparto. }\end{array}$ & $\begin{array}{l}\text { Ensaio clínico } \\
\text { randomizado }\end{array}$ & $\begin{array}{l}\text { O protocolo do estudo Terapias } \\
\text { Complementares para o Trabalho de Parto e } \\
\text { Nascimento reduziu significativamente o uso } \\
\text { de peridural e cesárea. }\end{array}$ \\
\hline $\begin{array}{l}\text { Efeitos da aromaterapia com } \\
\text { rosa damascena na dor e na } \\
\text { ansiedade do parto de } \\
\text { mulheres nulíparas durante a } \\
\text { primeira fase do trabalho de } \\
\text { parto }\end{array}$ & $\begin{array}{l}\text { HAMDAMIAN S, } \\
\text { et al., } 2018\end{array}$ & $\begin{array}{c}\text { Avaliar os efeitos da aromaterapia } \\
\text { com Rosa damascena sobre a dor } \\
\text { e a ansiedade na primeira fase do } \\
\text { trabalho de parto em mulheres } \\
\text { nulíparas. }\end{array}$ & $\begin{array}{l}\text { Ensaio clínico } \\
\text { randomizado }\end{array}$ & $\begin{array}{c}\text { A intensidade da dor no grupo que recebeu } \\
\text { aromaterapia com R. damascena foi } \\
\text { significativamente menor do que no grupo } \\
\text { controle após o tratamento em cada avaliação } \\
\text { da dor. }\end{array}$ \\
\hline
\end{tabular}




\begin{tabular}{|c|c|c|c|c|}
\hline Título & Autor/ano & Objetivo & Tipo de estudo & Resultado \\
\hline $\begin{array}{l}\text { Bola de parto ou terapia de } \\
\text { calor? Um ensaio clínico } \\
\text { randomizado para comparar } \\
\text { a eficácia do uso da bola de } \\
\text { parto com a terapia de calor } \\
\text { perineal no sacro no controle } \\
\text { da dor do parto }\end{array}$ & $\begin{array}{l}\text { TAAVONI S, et } \\
\text { al., } 2016\end{array}$ & $\begin{array}{c}\text { Investigar os efeitos de dois } \\
\text { métodos não farmacológicos, como } \\
\text { a bola de parto e a terapia térmica, } \\
\text { no alívio da dor do parto. }\end{array}$ & $\begin{array}{l}\text { Ensaio clínico } \\
\text { randomizado }\end{array}$ & $\begin{array}{c}\text { O escore médio da intensidade da dor no } \\
\text { grupo de terapia térmica foi menor do que no } \\
\text { grupo controle após a intervenção. Além disso, } \\
\text { houve diferenças significativas entre os } \\
\text { escores de dor no grupo da bola de parto } \\
\text { depois de todos os três momentos } \\
\text { investigados em comparação ao grupo de } \\
\text { controle. }\end{array}$ \\
\hline $\begin{array}{l}\text { Efeito da mudança de } \\
\text { posição e massagem nas } \\
\text { costas na percepção da dor } \\
\text { durante a primeira fase do } \\
\text { parto }\end{array}$ & $\begin{array}{l}\text { ALI SAK e } \\
\text { AHMED HM, } \\
2018\end{array}$ & $\begin{array}{c}\text { Identificar o impacto da mudança } \\
\text { de posição ou da massagem nas } \\
\text { costas na percepção da dor } \\
\text { durante a primeira fase do trabalho } \\
\text { de parto. }\end{array}$ & $\begin{array}{l}\text { Estudo quase } \\
\text { experimental }\end{array}$ & $\begin{array}{l}\text { A massagem nas costas pode ser uma } \\
\text { abordagem mais eficaz para o controle da dor } \\
\text { do que a mudança de posição durante o } \\
\text { primeiro estágio do trabalho de parto. }\end{array}$ \\
\hline $\begin{array}{l}\text { Efeitos da massagem lombar } \\
\text { na percepção da dor do } \\
\text { parto e na satisfação }\end{array}$ & $\begin{array}{l}\text { ERDOGAN SU, et } \\
\text { al., } 2017\end{array}$ & $\begin{array}{l}\text { Avaliar o efeito da massagem } \\
\text { lombar na percepção da dor do } \\
\text { parto e do parto. }\end{array}$ & $\begin{array}{l}\text { Estudo-controle } \\
\text { experimental }\end{array}$ & $\begin{array}{l}\text { A massagem lombar tem um impacto } \\
\text { significativo na redução da dor do parto e no } \\
\text { aumento da satisfação com o parto. }\end{array}$ \\
\hline $\begin{array}{l}\text { Efeitos da acupuntura } \mathrm{LI}-4 \text { e } \\
\text { SP-6 na dor do parto, nível } \\
\text { de cortisol e duração do } \\
\text { parto }\end{array}$ & $\begin{array}{l}\text { ASADI N, et al., } \\
2015\end{array}$ & $\begin{array}{l}\text { Investigar os efeitos da acupuntura } \\
\text { na dor do parto, nível de cortisol } \\
\text { sérico e duração do parto. }\end{array}$ & $\begin{array}{l}\text { Ensaio clínico } \\
\text { randomizado }\end{array}$ & $\begin{array}{l}\text { Não foram observadas variações significativas } \\
\text { nos escores de dor ou níveis de cortisol sérico } \\
\text { entre os dois grupos de controle. No entanto, a } \\
\text { duração do trabalho de parto foi } \\
\text { significativamente menor no grupo que } \\
\text { recebeu acupuntura real. }\end{array}$ \\
\hline $\begin{array}{l}\text { Efeito da acupressão LI4 e } \\
\text { BL32 na dor do parto e no } \\
\text { resultado do parto na } \\
\text { primeira fase do trabalho de } \\
\text { parto em mulheres } \\
\text { primíparas: um ensaio clínico } \\
\text { randomizado }\end{array}$ & $\begin{array}{l}\text { OZGOLI G, et al., } \\
2016\end{array}$ & $\begin{array}{l}\text { Examinar e comparar o efeito da } \\
\text { acupressão L14 e BL32 entre si e } \\
\text { com o grupo de controle na dor do } \\
\text { parto e nos resultados do parto. }\end{array}$ & $\begin{array}{l}\text { Ensaio clínico } \\
\text { randomizado }\end{array}$ & $\begin{array}{c}\text { A redução da dor foi significativamente maior } \\
\text { nos grupos LI4 e BL32 em comparação com o } \\
\text { controle. Além disso, a acupressão no ponto } \\
\text { BL32 foi superior ao ponto LI4 no alívio da dor } \\
\text { na primeira e na segunda, mas não na terceira } \\
\text { intervenção. }\end{array}$ \\
\hline
\end{tabular}




\begin{tabular}{|c|c|c|c|c|}
\hline Título & Autor/ano & Objetivo & Tipo de estudo & Resultado \\
\hline $\begin{array}{l}\text { Efeitos da eletroacupuntura } \\
\text { na redução da dor do parto e } \\
\text { complicações no processo } \\
\text { de analgesia do parto de } \\
\text { analgesia combinada raqui- } \\
\text { peridural com analgesia } \\
\text { peridural controlada pelo } \\
\text { paciente }\end{array}$ & $\begin{array}{l}\text { XIAO J, et al., } \\
2019\end{array}$ & $\begin{array}{c}\text { Comparar os escores de dor e as } \\
\text { taxas de complicações no processo } \\
\text { de analgesia do parto entre os dois } \\
\text { grupos. }\end{array}$ & $\begin{array}{l}\text { Ensaio clínico } \\
\text { randomizado }\end{array}$ & $\begin{array}{l}\text { Constatou-se que a eletroacupuntura auxilia } \\
\text { na redução da dor do parto. }\end{array}$ \\
\hline $\begin{array}{l}\text { O efeito da acupressão na } \\
\text { dor do parto e a duração do } \\
\text { trabalho de parto quando } \\
\text { aplicada ao ponto SP6: } \\
\text { ensaio clínico randomizado }\end{array}$ & $\begin{array}{l}\text { TURKMEN H e } \\
\text { TURFAN EÇ, } \\
2020\end{array}$ & $\begin{array}{l}\text { Determinar o efeito da acupressão } \\
\text { na primeira fase da dor do parto e } \\
\text { na duração. }\end{array}$ & $\begin{array}{l}\text { Estudo } \\
\text { experimental, } \\
\text { randomizado }\end{array}$ & $\begin{array}{l}\text { A dor do parto foi menor e a duração do } \\
\text { trabalho de parto foi menor para o grupo } \\
\text { experimental. A aplicação de acupressão em } \\
\text { SP6 mostrou ter um efeito positivo no parto, } \\
\text { reduzindo a dor. }\end{array}$ \\
\hline $\begin{array}{l}\text { O efeito da pressão de gelo } \\
\text { aplicada no intestino grosso } \\
4 \text { na dor do parto e no } \\
\text { processo de parto }\end{array}$ & $\begin{array}{l}\text { YILDIRIM E, et } \\
\quad \text { al., } 2018\end{array}$ & $\begin{array}{l}\text { Identificar o efeito da massagem } \\
\text { com gelo de curta duração ( } 40 \text { min) } \\
\text { e longa duração ( } 80 \text { min) aplicada } \\
\text { no ponto de acupressão do } \\
\text { Intestinal Grosso } 4 \text { (LI4) no } \\
\text { processo de trabalho de parto e na } \\
\text { percepção do dor de parto. }\end{array}$ & $\begin{array}{l}\text { Estudo } \\
\text { experimental }\end{array}$ & $\begin{array}{c}\text { Os resultados mostram que a massagem com } \\
\text { gelo aplicada na região LI4 foi eficaz aos } 80 \\
\text { minutos. }\end{array}$ \\
\hline $\begin{array}{l}\text { Efeitos da massagem e da } \\
\text { acupressão no alívio da dor } \\
\text { do parto, na redução do } \\
\text { tempo do parto e no } \\
\text { aumento da satisfação no } \\
\text { parto }\end{array}$ & $\begin{array}{l}\text { GONENÇ IM e } \\
\text { TERZIOGLU F, } \\
2020\end{array}$ & $\begin{array}{l}\text { Comparar os efeitos da massagem } \\
\text { e da acupressão no manejo da dor } \\
\text { relacionada ao parto. }\end{array}$ & $\begin{array}{l}\text { Ensaio clínico } \\
\text { randomizado }\end{array}$ & $\begin{array}{l}\text { A aplicação dupla de massagem e acupressão } \\
\text { é relativamente mais eficaz do que qualquer } \\
\text { terapia aplicada sozinha e que a massagem é } \\
\text { mais eficaz do que a acupressão. }\end{array}$ \\
\hline $\begin{array}{l}\text { A eficácia da intervenção de } \\
\text { hipnose para o parto: um } \\
\text { estudo experimental }\end{array}$ & $\begin{array}{l}\text { BEEVI Z, et al., } \\
\qquad 2017\end{array}$ & $\begin{array}{l}\text { Comparar as diferenças entre os } \\
\text { grupos experimental }(n=23) \text { e } \\
\text { controle }(n=22) \text { em variáveis } \\
\text { específicas medidas durante o } \\
\text { trabalho de parto. }\end{array}$ & $\begin{array}{l}\text { Ensaio clínico } \\
\text { randomizado }\end{array}$ & $\begin{array}{l}\text { Os participantes do grupo experimental } \\
\text { receberam a intervenção de hipnose, } \\
\text { enquanto os do grupo de controle receberam } \\
\text { apenas cuidados pré-natais de rotina. As } \\
\text { participantes do grupo experimental relataram } \\
\text { níveis mais elevados de dor imediatamente } \\
\text { antes, durante e imediatamente após o parto, } \\
\text { entretanto o uso de petidina durante o trabalho } \\
\text { de parto foi significativamente menor do que } \\
\text { as participantes do grupo controle. }\end{array}$ \\
\hline
\end{tabular}




\begin{tabular}{|c|c|c|c|c|}
\hline Título & Autor/ano & Objetivo & Tipo de estudo & Resultado \\
\hline $\begin{array}{l}\text { O efeito da acupressão } \\
\text { aplicada ao ponto LI4 nas } \\
\text { dores de parto percebidas }\end{array}$ & $\begin{array}{l}\text { HAMLACI Y e } \\
\text { YAZICI S, } 2017\end{array}$ & $\begin{array}{l}\text { Detectar o efeito da acupressão } \\
\text { aplicada ao Ponto LI4 na } \\
\text { percepção das dores de parto. }\end{array}$ & $\begin{array}{l}\text { Estudo } \\
\text { experimental } \\
\text { randomizado }\end{array}$ & $\begin{array}{c}\text { A aplicação de acupressão ao Ponto LI4 foi } \\
\text { considerada eficaz na redução da percepção } \\
\text { das dores do parto e no encurtamento do } \\
\text { trabalho de parto. }\end{array}$ \\
\hline $\begin{array}{c}\text { Uso de técnicas } \\
\text { farmacológicas e não } \\
\text { farmacológicas de controle } \\
\text { da dor do parto e sua relação } \\
\text { com os resultados do parto } \\
\text { materno e infantile: exame } \\
\text { de uma amostra } \\
\text { nacionalmente } \\
\text { representative de } 1835 \\
\text { mulheres grávidas }\end{array}$ & $\begin{array}{c}\text { ADAMS J, et al., } \\
2015\end{array}$ & $\begin{array}{l}\text { Investigar o uso por mulheres de } \\
\text { técnicas farmacológicas e não } \\
\text { farmacológicas de manejo da dor } \\
\text { do parto. }\end{array}$ & $\begin{array}{c}\text { Estudo } \\
\text { experimental }\end{array}$ & $\begin{array}{c}\text { A análise identificou o uso de água pelas } \\
\text { mulheres para o controle da dor do parto como } \\
\text { uma redução da probabilidade de seu bebê } \\
\text { ser admitido em berçário de cuidados } \\
\text { especiais e o uso de técnicas de respiração e } \\
\text { massagem para controle da dor aumentou a } \\
\text { probabilidade de mulheres continuarem } \\
\text { amamentando. }\end{array}$ \\
\hline $\begin{array}{l}\text { Experiências de mulheres } \\
\text { dinamarquesas com a } \\
\text { técnica de rebozo durante o } \\
\text { trabalho de parto: um estudo } \\
\quad \text { exploratório qualitativo }\end{array}$ & $\begin{array}{l}\text { IVERSEN ML, et } \\
\text { al., } 2017\end{array}$ & $\begin{array}{l}\text { Explorar as experiências de } \\
\text { mulheres com a técnica do rebozo } \\
\text { durante o trabalho de parto. }\end{array}$ & Estudo qualitativo & $\begin{array}{c}\text { As mulheres vivenciaram que a técnica com o } \\
\text { rebozo criava sensações corporais que } \\
\text { diminuem a dor. }\end{array}$ \\
\hline $\begin{array}{l}\text { Efeito da música no trabalho } \\
\text { de parto e no parto em } \\
\text { gestações únicas de } \\
\text { nulíparas: um ensaio clínico } \\
\text { randomizado }\end{array}$ & $\begin{array}{l}\text { BUGLIONE A, et } \\
\text { al., } 2020\end{array}$ & $\begin{array}{l}\text { Testar a hipótese de que em } \\
\text { mulheres nulíparas com gravidez } \\
\text { única a termo, ao ouvir música } \\
\text { reduziria o nível de dor durante o } \\
\text { trabalho de parto. }\end{array}$ & $\begin{array}{l}\text { Ensaio clínico } \\
\text { randomizado }\end{array}$ & $\begin{array}{l}\text { Música durante o trabalho de parto e parto foi } \\
\text { associada a uma diminuição da dor em } 1 \mathrm{~h} \\
\text { após o parto. Evidenciando que em mulheres } \\
\text { nulíparas com gravidez única a termo, ouvir } \\
\text { música reduz o nível de dor. }\end{array}$ \\
\hline $\begin{array}{c}\text { Auto hipnose para } \\
\text { tratamento da dor intraparto } \\
\text { em mulheres grávidas } \\
\text { nulíparas: um ensaio clínico } \\
\text { randomizado e controlado de } \\
\text { eficácia clínica }\end{array}$ & $\begin{array}{l}\text { DOWNE S, et al., } \\
2015\end{array}$ & $\begin{array}{l}\text { Estabelecer o efeito da auto } \\
\text { hipnose pré-natal em grupo para } \\
\text { mulheres nulíparas no uso de } \\
\text { peridural intraparto. }\end{array}$ & $\begin{array}{l}\text { Ensaio clínico } \\
\text { randomizado }\end{array}$ & $\begin{array}{l}\text { A alocação de sessões de treinamento de auto } \\
\text { hipnose nos grupos não reduziu } \\
\text { significativamente o uso de analgesia peridural } \\
\text { intraparto ou as dores. }\end{array}$ \\
\hline
\end{tabular}




\begin{tabular}{|c|c|c|c|c|}
\hline Título & Autor/ano & Objetivo & Tipo de estudo & Resultado \\
\hline $\begin{array}{l}\text { Implementação de um } \\
\text { protocolo de hidroterapia } \\
\text { para melhorar o controle da } \\
\text { dor pós-parto }\end{array}$ & $\begin{array}{l}\text { BATTEN M, et al., } \\
2017\end{array}$ & $\begin{array}{l}\text { Implementar um protocolo de } \\
\text { hidroterapia no período pós-parto } \\
\text { inicial para melhorar o controle da } \\
\text { dor para mulheres que optam por } \\
\text { um parto não medicamentoso. }\end{array}$ & $\begin{array}{l}\text { Estudo } \\
\text { experimental }\end{array}$ & $\begin{array}{l}\text { Em mulheres que usaram o banho, houve uma } \\
\text { redução significativa nos escores de dor. }\end{array}$ \\
\hline $\begin{array}{l}\text { loga pré-natal: efeitos no } \\
\text { alívio da dor do parto e } \\
\text { resultados do parto }\end{array}$ & $\begin{array}{l}\text { BOLANTHAKODI } \\
\text { C, et al., } 2018\end{array}$ & $\begin{array}{l}\text { Testar a hipótese de que os } \\
\text { exercícios de ioga pré-natais são } \\
\text { eficazes para aliviar a dor do parto. }\end{array}$ & $\begin{array}{l}\text { Ensaio clínico } \\
\text { randomizado }\end{array}$ & $\begin{array}{l}\text { A tolerância à dor foi melhor no grupo de } \\
\text { estudo que realizou a ioga, evidenciando que } \\
\text { ela é eficaz no alívio da dor do parto. }\end{array}$ \\
\hline $\begin{array}{l}\text { Eficácia dos exercícios } \\
\text { respiratórios durante a } \\
\text { segunda fase de trabalho de } \\
\text { parto na dor e duração do } \\
\text { parto: um ensaio clínico } \\
\text { randomizado }\end{array}$ & $\begin{array}{l}\text { YUKSEL H, et al., } \\
2017\end{array}$ & $\begin{array}{l}\text { Determinar se os exercícios } \\
\text { respiratórios para gestantes } \\
\text { durante o segundo estágio do } \\
\text { trabalho de parto têm efeitos } \\
\text { benéficos sobre a dor materna. }\end{array}$ & $\begin{array}{l}\text { Ensaio clínico } \\
\text { randomizado }\end{array}$ & $\begin{array}{l}\text { Exercícios respiratórios com inspiração e } \\
\text { expiração profundas em gestantes são } \\
\text { eficazes na redução da percepção da dor do } \\
\text { parto. }\end{array}$ \\
\hline
\end{tabular}

Fonte: França GSD, et al., 2021. 
Conforme disposto, o ano com maior número de publicações foi o de 2017, seguido dos anos de 2018 e 2016. Entretanto, existe uma variedade de publicações ao decorrer dos anos, o que implica dizer que estudos sobre a temática são publicados continuamente, fato importante para a atualização do assunto em questão.

O tipo de estudo mais prevalente foi o ensaio clínico randomizado, sendo que dos 30 artigos que foram selecionados, 19 destes são ensaio clínico, o que pode ser justificado pelo fato desse tipo de estudo ser utilizado para comparar o efeito e o valor de uma intervenção em dois grupos, comparando um grupo com o outro, e esse é justamente o objetivo da presente pesquisa, identificar quais são os métodos não farmacológicos utilizados para alívio da dor durante o trabalho de parto e no parto e para que se possa identificar quais métodos são mais eficazes é necessário comparar o grupo de parturientes que não utilizaram nenhum método com o grupo que utilizou, e assim analisar a eficácia dos mesmos.

Dos 30 artigos que compuseram a amostra desta pesquisa, todos tiveram como população de pesquisa gestantes que estavam em trabalho de parto ou parto e todos investigaram a utilização de algum método não farmacológico durante esse processo.

Após categorização inicial dos artigos que compuseram a amostra final, os mesmos foram organizados quanto aos métodos não farmacológicos utilizados para alívio da dor durante o trabalho de parto e parto (Tabela 1). Os métodos não farmacológicos foram organizados por ordem crescente de acordo com a quantidade de artigos em que são citados.

Tabela 1 - Caracterização dos artigos quanto aos métodos não farmacológicos utilizados para alívio da dor durante o trabalho de parto e parto.

\begin{tabular}{|lcc|}
\hline \multicolumn{1}{c}{ Métodos não farmacológicos } & Número de artigos & $\%$ \\
\hline Massagem & 7 & 23,3 \\
Acupressão & 5 & 16,7 \\
Utilização da bola suíça & 3 & 10 \\
Aromaterapia & 3 & 10 \\
Banho de chuveiro e/ou banho quente & 2 & 6,7 \\
Exercício perineal & 2 & 6,7 \\
Musicoterapia & 2 & 6,7 \\
Hipnose & 2 & 6,7 \\
loga durante a gestação & 2 & 6,7 \\
Acupuntura & 1 & 3,3 \\
Eletroacupuntura & 1 & 3,3 \\
Técnicas de respiração & 1 & 3,3 \\
Técnica de reflexologia & 1 & 3,3 \\
Auriculoterapia & 1 & 3,3 \\
Técnica do rebozo & 1 & 3,3 \\
Terapia de calor & 1 & 3,3 \\
Mudança de posição & 1 & 3,3 \\
\hline
\end{tabular}

Fonte: França GSD, et al., 2021.

Com relação aos métodos não farmacológicos utilizados para alívio da dor durante o trabalho de parto e no parto, foi possível identificar que os métodos citados pelos estudos foram auriculoterapia, banho de chuveiro e/ou banho quente, utilização da bola e exercícios perineais realizados na bola, acupressão no ponto sanyinjiao, no ponto LI4, no ponto BL32 e no ponto SP6, acupuntura, eletroacupuntura, aromaterapia com essência de lavanda e com rosa damascena, musicoterapia, massagem com gelo no ponto Hoku ou na região LI4, massagem sacral e lombar, técnica de reflexologia, yoga durante a gestação, hipnose, técnicas de respiração com inspiração e expiração profunda, técnica do rebozo e associação de técnicas como massagem e acupressão juntos ou banho juntamente com a bola.

\section{DISCUSSÃO}

O método não farmacológico mais mencionado pelos estudos selecionados foram as massagens, diversos estudos corroboram o que foi constatado por este estudo. Uma pesquisa randomizada realizada com 60 
primíparas em trabalho de parto, avaliou o efeito da massagem durante as contrações uterinas, sendo constatado que a massagem pode diminuir consideravelmente a intensidade da dor. Além disso, a massagem pode ser feita em qualquer região do corpo em que a parturiente relate desconforto e também pode ser associada com outros métodos, podendo ser aplicada pelo acompanhante ou pelos profissionais (CHANG MY, et al., 2006).

Segundo o estudo de Chang MY, et al. (2006), os resultados indicam que apesar da massagem não alterar as características das dores sentidas pelas parturientes durante o trabalho de parto, ela pode realmente diminuir a intensidade da dor durante o parto na primeira e segunda fase da dilatação cervical e pode acalmar a gestante, reduzindo os níveis de ansiedade e estresse.

Outros métodos citados pelos estudos foram as massagens com gelo no ponto Hoku, na região sacral e na região lombar. O ponto Hoku, também chamado de ponto L14 é um ponto localizado entre o polegar e o dedo indicador, que auxilia no alívio das dores durante o parto e pode ser utilizado junto com gelo, sendo um método barato e facilmente disponível (DEHCHESHMEH FS e RAFIEI H, 2015).

Outro método muito citado pelos estudos selecionados foi a acupressão, no qual foram mencionados a acupressão no ponto sanyinjiao (SP6), no ponto L14 e no ponto BL32. O que corrobora com ensaios controlados e randomizados realizados em alguns países da Ásia e do Oriente Médio no qual foi evidenciado os efeitos positivos da acupressão sobre a dor durante o trabalho de parto e parto. Nesses estudos foram identificados três pontos, entre eles o ponto SP6, caracterizado por possuir forte influência nos órgãos reprodutivos (HAN JS, 2003).

O ponto SP6 está localizado no meridiano entre o baço e o pâncreas, quatro dedos acima do maléolo interno na parte frontal da tíbia e a acupressão nesse ponto possui a capacidade de controlar alguns aspectos dos órgãos reprodutivos, sendo um método não farmacológico útil para aliviar a dor de gestantes em trabalho de parto de maneira não invasiva (MAFETONI RR e SHIMO AKK, 2016).

A acupressão possui princípios iguais aos da acupuntura, no qual o objetivo é manter o equilíbrio energético nos diversos canais circulantes do corpo, conectados a algum órgão alvo, mas sem a utilização de agulhas. Os estímulos são realizados por meio das mãos e dedos em pontos específicos, proporcionando assim maior alívio da dor e um estado de relaxamento (HAN JS, 2003).

A bola suíça também é um método não farmacológico atuante no alívio de dores durante esse processo. Porém, Lopes TC, et al. (2003) em seu estudo controle relataram que a bola é um recurso que deve ser usado com o intuito de auxiliar a parturiente na adoção da postura vertical de maneira confortável. Entretanto acrescenta afirmando que através da postura vertical e da movimentação é possível reduzir a dor da parturiente.

Segundo Melo PDS, et al. (2020) a bola suíça é um método não farmacológico utilizado durante o trabalho de parto com o intuito de realizar exercícios perineais, sendo uma maneira de estimular a dilatação cervical da parturiente, assim como a progressão do feto por meio da pelve e o relaxamento dos músculos perineais, ocasionando no alívio da dor.

A aromaterapia também foi mencionada por alguns estudos como um método não farmacológico que auxilia no alívio das dores durante o trabalho de parto e parto. Em consonância a isso, segundo Yazdkhasti M e Pirak A (2016), a aromaterapia tem ajudado as mulheres durante o trabalho de parto, por meio da utilização dos óleos essenciais. Os óleos são instáveis em sua forma bioquímica, evaporando rapidamente e dessa forma eles estimulam as células nervosas olfativas, ativando os receptores que atuam na frequência cardíaca, respiração e na resposta ao estresse.

Conforme constatado no estudo, as essências utilizadas para alívio das dores foram as de lavanda e as de rosa damascena. Durante a primeira fase do trabalho de parto devem ser utilizados óleos com propriedades calmantes, entre eles a lavanda, e camomila, o óleo de olíbano e a sálvia sclarea. Já na segunda fase do trabalho de parto, devem ser utilizados aromas mais fortes para promover sensação de força e aumentar as contrações, entre eles o óleo de jasmin. Ainda ressaltam que a aromaterapia deve ser utilizada 
cuidadosamente, pois a inalação prolongada dos óleos pode ocasionar dores de cabeça, náuseas, alergias e irritação na pele (YAZDKHASTI M e PIRAK A, 2016).

No que se refere ao banho de chuveiro, a água quente promove vasodilatação periférica, redistribuindo o fluxo sanguíneo, ocasionando o relaxamento muscular e exercendo influência positiva sobre a dor. Em concordância a isso, um ensaio clínico randomizado realizado com 100 parturientes que investigou o efeito do banho de chuveiro no alívio da dor durante o trabalho de parto evidenciou que o método é eficaz na diminuição da intensidade da dor durante a fase ativa da dilatação (DAVIM RMB, et al., 2008).

A hidroterapia, mais especificamente o banho quente, tem sido um método não farmacológico amplamente utilizado no decorrer do trabalho de parto, sendo bem aceito pelas parturientes. O banho proporciona conforto, relaxamento e melhora dos níveis de dor, ansiedade e estresse. Além disso, auxilia no aumento da dilatação cervical, na diminuição da pressão sanguínea e na redução do uso de analgésicos (MELO PDS, et al., 2020).

Segundo Davim RMB, et al. (2008), o conforto físico das parturientes é favorecido pela utilização das terapias complementares, entre elas o banho no chuveiro, a cromoterapia, a massagem, o uso da bola, musicoterapia e hidroterapia. Dessa maneira, essas técnicas necessitam ser implementadas dentro do ambiente da maternidade pelas enfermeiras, com o intuito de integrar essas terapias da assistência da enfermagem em gestantes no seu processo parturitivo.

Em determinadas situações, o uso de métodos invasivos e de elementos estimuladores de sentidos elevam a dor. Entretanto, a liberdade de movimento, a utilização do banho quente e de exercícios perineais com bola suíça, isolados ou combinados, estão associados ao conforto e a redução da dor (MELO PDS, et al., 2020).

A musicoterapia também é mencionada pelos estudos como um método que auxilia da diminuição da dor. Segundo um ensaio clínico randomizado não cego de grupo paralelo realizado em um centro de parto na Itália com mulheres nulíparas, a música durante o trabalho de parto e parto foi associada a uma redução da dor em uma hora pós-parto e também na redução do nível de ansiedade durante a fase ativa do trabalho de parto (DEHCHESHMEH FS e RAFIEI H, 2015).

Já o método de hipnose também foi citado como um método que auxilia na diminuição das dores do trabalho de parto, visto que ao mentalizar cenas positivas, como a imagem do filho nos braços ou momentos de felicidade e prazer, é possível aproveitar mais o trabalho de parto (BEEVI Z, et al., 2017). Entretanto, em discordância a isso, uma pesquisa de controle randomizado multi-método realizado com 680 mulheres nulíparas que não planejam cesárea eletiva, constatou que as sessões de treinamento de auto hipnose nos grupos não reduziu significativamente o uso de analgesia peridural intraparto ou as dores (DOWNE S, et al., 2015).

A acupuntura, assim como a eletroacupuntura também são mencionados por estudos como eficazes para diminuição da dor durante o trabalho de parto. A ação da acupuntura na área da analgesia tem sido tema de estudos, principalmente em experimentos no campo da neurofisiologia efetuados pelo Instituto de Pesquisas em Neurociências da Universidade de Pequim, Beijing, China. Esses estudos corroboram com o que foi constatado, demonstrando que a eletroacupuntura aciona o sistema supressor da dor por meio da estimulação das terminações nervosas, gerando a analgesia, amenizando as dores do parto (HAN JS, 2003).

Além disso, outros estudos também corroboram com esta pesquisa, relatando que a partir do retorno dado pelas puérperas, foi possível observar a eficácia do método, pois todas responderam positivamente com relação ao alívio da dor ou ausência de outros desconfortos (CHEROBIN F, et al., 2016; CHANG MY, et al., 2006).

Cherobin F, et al. (2016) afirmam que o interesse de estudar a acupuntura durante $o$ trabalho de parto e parto se dá pelos diversos benefícios que ela promove para mãe e filho, pois não altera os níveis de consciência materna, o que proporciona maior envolvimento da mesma durante todo processo, não exclui a utilização de outros métodos de analgesia e é uma alternativa economicamente viável, econômica, segura e sem efeitos colaterais.

Por conseguinte, outro método não farmacológico citado foram os exercícios respiratórios e esses exercícios ajudam a diminuir a percepção dolorosa, melhorando os níveis de saturação materna, promovendo 
relaxamento e reduzindo a ansiedade. Indo de encontro ao que foi evidenciado, Boing L, et al. (2007) ao realizarem um estudo randomizado com 40 primigestas, no qual o grupo experimental realizou o método respiratório diafragmático de maneira lenta e profunda, constataram que houve diminuição da intensidade dolorosa, assim como aumento da saturação de oxigênio.

Entretanto, em discordância a isso, um estudo que avaliou o efeito das técnicas de respiração e de relaxamento sobre a dor e a ansiedade na parturição por meio de um estudo randomizado e controlado com 36 parturientes, constatou-se que as técnicas de respiração utilizadas não diminuíram a intensidade da dor, mas reduziram os níveis de ansiedade (ALMEIDA NAM, et al., 2005). Essa diferença de resultados pode ter se dado devido ao fato do estudo de Boing $L$, et al. (2007) ter utilizado o método respiratório diafragmático de maneira lenta e profunda e o estudo de Almeida NAM, et al. (2005) ter utilizado técnicas gerais de relaxamento.

Além disso outros métodos citados no presente estudo também são mencionados em outras pesquisas como sendo eficazes para reduzir a dor da parturiente durante o trabalho de parto e parto, entre eles a técnica de reflexologia, a ioga durante a gestação, a auriculoterapia, a técnica do rebozo, a terapia de calor e a mudança de posição (MAFETONI RR, et al., 2019; MOGHIMI-HANJANI S, et al., 2015; JAHDI F, et al., 2017; TAAVONI S, et al., 2016; ALI SAK e AHMED HM, 2018; IVERSEN ML, et al., 2017; BOLANTHAKODI C, et al., 2018).

\section{CONSIDERAÇÕES FINAIS}

Diante do exposto identificou-se quais são os métodos não farmacológicos utilizados para alívio da dor durante o trabalho de parto e no parto, entre eles: auriculoterapia, banho de chuveiro, utilização da bola, acupressão, acupuntura, aromaterapia, musicoterapia, massagem, técnica de reflexologia, yoga, hipnose, técnicas de respiração e técnica do rebozo. Foi possível evidenciar através desse estudo a eficácia de métodos não farmacológicos para reduzir a dor durante o trabalho de parto e parto, sendo eles diversificados e podendo ser aplicados de diversas maneiras e com baixo custo. Além disso, foi possível também constatar que além do alívio da dor, estes métodos proporcionam relaxamento e diminuem a ansiedade das parturientes, sendo métodos seguros e efetivos.

\section{REFERÊNCIAS}

1. ADAMS J, et al. Use of pharmacological and non-pharmacological labour pain management techniques and their relationship to maternal and infant birth outcomes: examination of a nationally representative sample of 1835 pregnant women. Midwifery, 2015; 31(4): 458-63.

2. ALI SAK, AHMED HM. Effect of Change in Position and Back Massage on Pain Perception during First Stage of Labor. Pain Manag Nurs., 2018; 19(3): 288-294.

3. ALMEIDA NAM, et al. Utilização de técnicas de respiração e relaxamento para alívio de dor e ansiedade no processo de parturição. Rev Lat Am Enferm., 2005; 13(1): 52-8.

4. ASADI N, et al. Effects of LI-4 and SP-6 Acupuncture on Labor Pain, Cortisol Level and Duration of Labor. J Acupunct Meridian Stud., 2015; 8(5): 249-54.

5. BATTEN M, et al. Implementation of a Hydrotherapy Protocol to Improve Postpartum Pain Management. J Mi dwifery Womens Health, 2017; 62(2): 210-214.

6. BEEVI Z, et al. The Effectiveness of Hypnosis Intervention for Labor: An Experimental Study. Am J Clin Hypn., 2017; 60(2): 172-191.

7. BOING L, et al. Uso de técnica respiratória para analgesia no parto. FEMINA, 2007; 35(1): 41-46.

8. BOLANTHAKODI C, et al. Prenatal Yoga: Effects on Alleviation of Labor Pain and Birth Outcomes. J Altern Complement Med., 2018; 24(12): 1181-1188.

9. BOTELHO LLR, et al. The integrative review method in organizational studies. Rev Eletr Gestão Soc., 2011; 5(11): 121-136.

10. BUGLIONE A, et al. Effect of music on labor and delivery in nulliparous singleton pregnancies: a randomized clinical trial. Arch Gynecol Obstet., 2020; 301(3): 693-698.

11. CAVALCANTI ACV, et al. Terapias complementares no trabalho de parto: ensaio clínico randomizado. Rev. Gaúcha Enferm., 2019; 40(e20190026): 1-9.

12. ÇEVIK SA, KARADUMAN S. The effect of sacral massage on labor pain and anxiety: A randomized controlled trial. Jpn J Nurs Sci., 2019; 17(1): e12272.

13. CHANG MY, et al. A Comparison of Massage Effects on Labor Pain Using the McGill Pain Questionnaire. J Nurs Res., 2006; 14(3): 190-7. 
14. CHEROBIN F, et al. Acupuntura e auriculoterapia como métodos não farmacológicos de alívio da dor no processo de parturição. Cogitare Enferm., 2016; 21(3): 1-8.

15. DAVIM RMB, et al. Banho de chuveiro como estratégia não farmacológica no alívio da dor em parturientes. Rev Eletr Enferm., 2008; 10(3): 600-9.

16. DEHCHESHMEH FS, RAFIEI H. Complementary and alternative therapies to relieve labor pain: A comparative study between music therapy and Hoku point ice massage. Complement Ther Clin Pract., 2015; 21(4): 229-32.

17. DOWNE S, et al. Self-hypnosis for intrapartum pain management in pregnant nulliparous women: a randomised controlled trial of clinical effectiveness. BJOG, 2015; 122(9): 1226-34.

18. ERDOGAN SU, et al. Effects of low back massage on perceived birth pain and satisfaction. Complement Ther Clin Pract., 2017; 28: 169-175.

19. GONENÇ IM, TERZIOGLU F. Effects of Massage and Acupressure on Relieving Labor Pain, Reducing Labor Time, and Increasing Delivery Satisfaction. J Nurs Res., 2020; 28(1): e68.

20. HALL JE. Guyton and Hall textbook medical of physiology. Philadelphia: Saunders Elsevier, 2015.

21. HAMDAMIAN S, et al. Effects of aromatherapy with Rosa damascena on nulliparous women's pain and anxiety of labor during first stage of labor. J Integr Med., 2018; 16(2): 120-125.

22. HAMLACI Y, YAZICI S. The Effect of Acupressure Applied to Point LI4 on Perceived Labor Pains. Holist Nurs Pract., 2017; 31(3): 167-176.

23. HAN JS. Acupuncture: neuropeptide release produced by electrical stimulation of different frequencies. Trends in Neurosciences, 2003; 26(1): 17-22.

24. IVERSEN ML, et al. Danish women's experiences of the rebozo technique during labour: A qualitative explorative study. Sex Reprod Healthc., 2017; 11: 79-85.

25. JAHDI F, et al. Yoga during pregnancy: The effects on labor pain and delivery outcomes (A randomized controlled trial). Complement Ther Clin Pract., 2016; 27:1-4.

26. LEVETT KM, et al. Complementary therapies for labour and birth study: a randomised controlled trial of antenatal integrative medicine for pain management in labour. BMJ Open, 2016; 6(7): e010691.

27. LOPES TC, et al. O uso da bola do nascimento na promoção da posição vertical em primíparas durante o trabalho de parto. Rev Min Enferm., 2003; 7(2): 134-9.

28. MAFETONI RR, et al. Effectiveness of auricular therapy on labor pain: a randomized clinical trial. Texto contexto enferm., 2019; 28(e20180110): 1-14.

29. MAFETONI RR, SHIMO AKK. The effects of acupressure on labor pains during child birth: randomized clinical trial. Rev. Latino-Am., 2016; 24(e2738): 1-8.

30. MAZONI SR, et al. Clinical validation of the nursing diagnosis labor pain. Rev Latino-Am Enfermagem, 2013; 21(Spec): 88-96.

31. MELO PDS, et al. Parâmetros maternos e perinatais após intervenções não farmacológicas: um ensaio clínico randomizado controlado. Acta paul. Enferm., 2020; 33(eAPE20190136): 1-9.

32. MOGHIMI-HANJANI S, et al. The Effect of Foot Reflexology on Anxiety, Pain, and Outcomes of the Labor in Primigravida Women. Acta Med Iran., 2015; 53(8): 507-11.

33. OZGOLI G, et al. Effect of LI4 and BL32 acupressure on labor pain and delivery outcome in the first stage of labor in primiparous women: A randomized controlled trial. Complement Ther Med., 2016; 29: 175-180.

34. TAAVONI S, et al. Birth ball or heat therapy? A randomized controlled trial to compare the effectiveness of birth ball usage with sacrum-perineal heat therapy in labor pain management. Complement Ther Clin Pract., 2016; 24: 99-102.

35. TANVISUT R, et al. Efficacy of aromatherapy for reducing pain during labor: a randomized controlled trial. Arch Gynecol Obstet., 2018; 297(5): 1145-1150.

36. TURKMEN H, TURFAN EÇ. The effect of acupressure on labor pain and the duration of labor when applied to the SP6 point: Randomized clinical trial. Jpn J Nurs Sci., 2020; 17(1): e12256.

37. XIAO J, et al. Effects of electroacupuncture on reducing labor pain and complications in the labor analgesia process of combined spinal-epidural analgesia with patient-controlled epidural analgesia. Arch Gynecol Obstet., 2019; 299(1): 123-128.

38. YAZDKHASTI M, PIRAK A. The effect of aromatherapy with lavender essence on severity of labor pain and duration of labor in primiparous women. Complement Ther Clin Pract., 2016; 25: 81-86.

39. YILDIRIM E, et al. The effect of ice pressure applied on large intestinal 4 on the labor pain and labor process. Complement Ther Clin Pract., 2018; 32: 25-31.

40. YUKSEL $\mathrm{H}$, et al. Effectiveness of breathing exercises during the second stage of labor on labor pain and duration: a randomized controlled trial. J Integr Med., 2017; 15(6): 456-461. 